\title{
EMERGING TRENDS IN ADAPTIVE COMPUTATION FOR MOBIQUITOUS SYSTEMS
}

\author{
Jesus Friginal \\ Research fellow at LAAS-CNRS, France
}

The future is in networked embedded systems. This affirmation is based on the principle that networked embedded systems will make possible the development of more intelligent living, work, entertainment, energy production systems and transport environments. It is estimated that the number of networked smart objects on a planetary scale will increase beyond 20 billion units by 2020 , thus promoting the emergence of a new type of network with ubiquitous nature called Internet of Things (IoT). As can be foreseen, no system in the future will run in isolation. IoT systems will be composed of myriads of computers, small devices, smart sensors and conventional computers ranging from laptops to servers, being inter-connected by fixed and/or wireless networks. Such systems will constitute an essential fabric of our society, as already witnessed by the dependence placed on current computing systems for almost all of our life activities. Among other specific features, what makes mobile and ubiquitous systems different and interesting from a research viewpoint is the interaction between mobile users, applications and devices and the intrinsic need of adaptation to changes in dynamic execution environments.
The areas addressed by this special issue include systems, applications, social networks, middleware, networking, data management and services, all with special focus on adaptive mobility and ubiquitous computing. We claim that this type of special issues is essential to shed some light in the future conception of mobiquitous systems. 